\title{
MEMORIA DE LLEGADAS E INGRESOS POR TURISMO EN CUBA EN EL LUSTRO 2015-2019
}

\section{MEMORY OF ARRIVALS AND REVENUES FOR TOURISM IN CUBA IN THE HALF A DECADE 2015-2019}

Héctor Ayala Castro, Ph.D.

Doctor en Ciencias Económicas (Cuba).

Profesor y Consultante de la Facultad de Turismo de la Universidad de la Habana, Cuba. hectorsalvadorayala@gmail.com

Yoan Hernández Flores, Lic. Licenciado en Turismo (Cuba). Profesor e Investigador de la Facultad de Turismo de la Universidad de la Habana, Cuba. yoan961122@gmail.com

\section{ARTÍCULO DE INVESTIGACIÓN}

Recibido: 14 de octubre de 2020

Aceptado: 11 de enero de 2021

\section{RESUMEN}

Para los gestores de las actividades vinculadas al sector del turismo, las tareas de planificación cobran mayor importancia en un contexto donde crece la demanda y varían las necesidades de los consumidores, por lo que se deben contar con bases de datos que permitan realizar pronósticos futuros. Cuba no está ajena a esta tendencia, es por ello que, con el objetivo de analizar el comportamiento de las llegadas y los ingresos por turismo en el lustro 2015 - 2019, al destino, se realiza la presente investigación. Con la combinación de diferentes métodos teóricos y empíricos se realiza un estudio de estos indicadores comparándolos con periodos anteriores, y con los principales destinos del Caribe Insular. El principal método utilizado es el análisis documental, pues se consultan varias fuentes de información de Cuba y del resto del mundo, con el fin de homogenizar los datos presentados, en la mayor medida posible. Finalmente, se muestra un análisis detallado que permite establecer la evolución del sector del turismo en la isla en este lustro, a partir de los indicadores seleccionados. De igual forma se muestran los cambios en el período analizado, así como algunas de sus consecuencias, sentando las bases para futuros estudios.

Palabras claves: Turismo, comportamiento de las llegadas, ingresos por turismo, Cuba. 


\section{ABSTRACT}

For managers of activities related to the tourism sector, planning tasks become more important in a context where demand is growing and consumer needs vary, so databases must be available that allow future forecasts to be made. Cuba is not alien to this trend, that is why, with the aim of analyzing the behavior of arrivals and tourism receipts in the five-year period 2015 - 2019, to the destination, this research is carried out. With the combination of different theoretical and empirical methods, a study of these indicators is carried out, comparing them with previous periods, and with the main destinations of the Insular Caribbean. The main method used is documentary analysis, since various sources of information from Cuba and the rest of the world are consulted, in order to homogenize the data presented, as much as possible. Finally, a detailed analysis is shown that allows establishing the evolution of the tourism sector on the island in this five-year period, based on the selected indicators. Likewise, the changes in the analyzed period are shown, as well as some of their consequences, laying the foundations for future studies.

Keywords: Tourism, behavior of arrivals, tourism receipts, Cuba.

\section{INTRODUCCIÓN}

El turismo ha sufrido un proceso de adaptación a lo largo de su historia. Varios investigadores afirman que la práctica de esta actividad se origina desde la época de los griegos, aunque fue a finales del siglo XIX (Ilamado siglo de la industrialización) y principios del siglo XX, cuando se consideró como actividad económica de gran relevancia, gracias a los avances industriales que facilitaron los traslados de un lugar a otro.

Para la Organización Mundial del Turismo (OMT, 2014), el turismo hace referencia "a los viajes y estancias que realizan las personas en lugares distintos a su entorno habitual, por un periodo mínimo de una noche de estadía y como máximo 365 días, por razones de ocio, negocios u otros motivos".

A su vez Kieffer (2018) enuncia que el turismo, ha sido un factor importante para el crecimiento de las economías locales, y un eje para el desarrollo de los territorios a nivel urbano y rural. Así, a nivel mundial el desarrollo turístico forma parte de las políticas, planes y programas de los países de la región y el mundo.

Los gestores del turismo, han enunciado y valoran como necesario el desarrollo de actividades de planificación dentro de las diferentes áreas del sector, la cual se basa en el establecimiento de objetivos o metas, y la elección de los medios más convenientes para alcanzarlos. 
Según Hernández (2020), elementos esenciales en la planificación turística son la selección de la información, el desarrollo de acciones con el fin de lograr el objetivo que se persigue, lo que implica conocerlo, evaluar la situación, considerar diferentes acciones que puedan realizarse y escoger la mejor alternativa. Dentro de ellos, los resultados estadísticos y las bases de datos con información que permita establecer comparaciones entre diferentes indicadores, cobran mayor protagonismo.

A nivel internacional, dentro de la planificación, conocer elementos como las llegadas de visitantes y turistas, y los ingresos generados por el turismo, son esenciales para evaluar el desempeño de los destinos turísticos.

En Cuba, se han desarrollado investigaciones como las presentadas por Ayala $(2013,2016)$, los cuales han contribuido a la organización y planificación de las entidades del sector, así como estudios desarrollados en las universidades en el país.

Estos estudios reflejan que, en la década de 90, del siglo pasado, Cuba priorizó el desarrollo del turismo internacional, donde se obtuvieron resultados sobresalientes. De igual forma los resultados alcanzados entre 2001 y 2014 fueron modestos, aunque se pueden considerar notables en el contexto en que se desarrollaron.

Si bien en Cuba, la Oficina Nacional de Información y Estadística (ONEI), confecciona estadísticas relativas al sector de turismo y con los principales indicadores, en ocasiones no se reflejan todos los datos necesarios, algunas informaciones no son similares a las que manejan los organismos especializados, en la base de datos presentes en la web existe carencia en cuanto a la sistematicidad de la información brindada, aparte que no se realiza un análisis de las estadísticas.

Esto se constituye en limitaciones a erradicar en vista de que los gestores del turismo cuenten con bases de datos lo más actualizadas posibles, las cuales contribuyan a las decisiones estratégicas a seguir en las organizaciones.

La presente investigación tiene el objetivo de analizar el comportamiento de las llegadas, y los ingresos por turismo en Cuba en el lustro 2015 - 2019, comparándolos con periodos anteriores, y con los principales destinos del Caribe Insular.

\section{METODOLOGÍA}

De acuerdo con Hernández, Fernández y Baptista (2014), la investigación se clasifica como descriptiva, pues en esta se describe la situación del turismo en el destino Cuba, en los años analizados, a través de los indicadores seleccionados. 
Además, es clasificada como de naturaleza cuantitativa y cualitativa, pues se utilizan también análisis matemáticos y descripciones cualitativas.

Dentro de los métodos teóricos, se utilizaron:

Análisis-síntesis: posibilita el procesamiento de la información a partir de la bibliografía consultada.

Histórico-lógico: permite el estudio del desarrollo de los indicadores, y a su vez establecer un análisis a partir de comportamientos anteriores.

En cuanto a los métodos empíricos se utilizó el análisis documental, pues una fuente muy valiosa de datos cualitativos fueron los documentos y materiales diversos que se consultaron, los que ayudaron a entender el fenómeno central de estudio. Se analizaron diferentes documentos con datos numéricos tanto de Cuba como de otros países del mundo. Destacan entre las fuentes fundamentales las de la ONEI y la OMT.

Todos los elementos anteriores, permitieron a los autores analizar e integrar la información existente, en aras de presentar un estudio completo de los indicadores seleccionados en el período de $2015-2019$.

\section{ANÁLISIS Y DISCUSIÓN DE LOS RESULTADOS}

Es válido señalar que, en este trabajo, se asumen los conceptos brindados OMT (2020) como son:

Llegadas de visitantes: se refieren a las personas que visitan el país por un periodo no superior a un año por ocio, recreo, negocio u otro motivo personal y no para ejercer actividad remunerada. Se divide en turistas (estancia superior a un día y e inferior a un año) y excursionistas (estancia inferior a 24 horas) que incluyen a pasajeros de cruceros, visitantes del día y tripulaciones.

Ingresos asociados al turismo internacional: los gastos efectuados en el país de acogida por los visitantes internacionales, incluido el pago de sus transportes internacionales a las compañías nacionales de transportación.

De igual manera, según lo expresado por ONEI (2019b), los ingresos por turismo son analizados como los ingresos que reportan las entidades turísticas y otras entidades que desarrollen servicios para el turismo internacional. 


\section{Llegadas de visitantes, turistas, mercados y motivaciones.}

Las llegadas de visitantes durante el periodo 2015 - 2019 crecen en los primeros tres años a igual nivel que en los mejores años del turismo en Cuba (períodos anteriores al 2015), pero en los dos años finales, crecen poco y decrecen, como puede observarse en la figura 1:

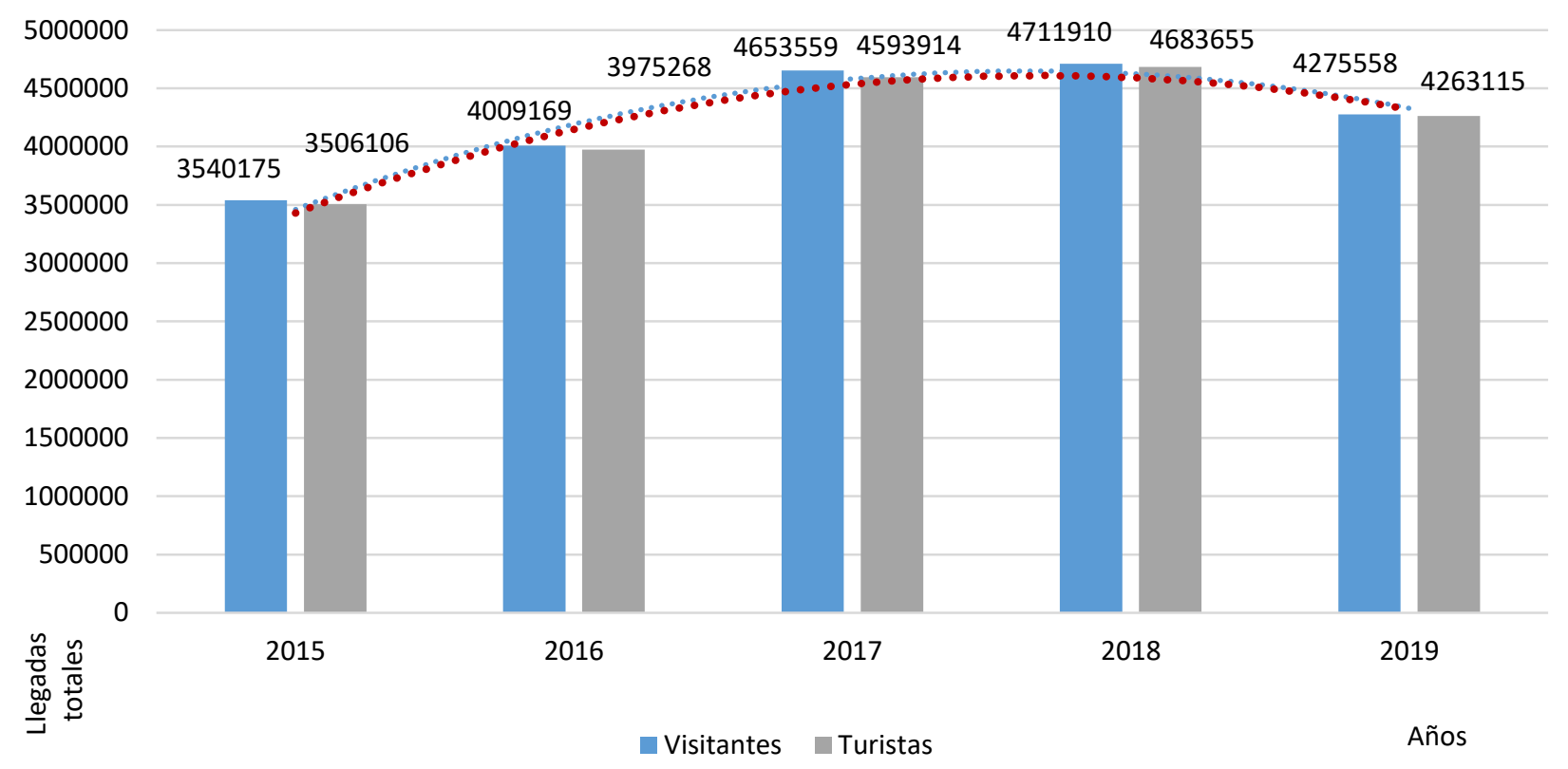

Figura 1. Llegada de visitantes y turistas a Cuba 2015-2019.

Fuente: Oficina Nacional de Estadísticas e Información (ONEI, 2019a).

En 2015, el crecimiento de visitantes (17,5 \%) se constituyó en uno de los más altos de todos los tiempos, incluso mayor que el registrado como promedio de crecimiento en la década dorada del 90 para el turismo cubano (17\%), elemento que resalta si se compara tanto con el crecimiento de 5,3\% que alcanzaron las llegadas de visitantes en el año anterior 2014, como con el 4,3\% de crecimiento medio anual de cinco años precedentes (2010 - 2014). El crecimiento de turistas fue mayor aún en 2015: 17,6 \%.

A partir de las declaraciones de los presidentes de Cuba y EE.UU., el 17 de diciembre de 2014 sobre la intención de entrar en un nuevo escenario de relaciones, se impulsaron en el 2015 las llegadas de visitantes a Cuba de forma impresionante desde EE.UU. (176,7 \% de crecimiento), pero también aumentaron notablemente las llegadas desde los mercados europeos, México, Argentina y la Comunidad Cubana en el Exterior (desde distintos países). De igual forma en este período existe un buen desenvolvimiento del fuerte mercado canadiense (Tabla 1). 
Tabla 1

Visitantes al destino Cuba de acuerdo a los principales mercados emisores.

\begin{tabular}{|c|c|c|c|c|c|c|c|c|c|c|}
\hline $\begin{array}{l}\text { Mercado } \\
\text { Emisor }\end{array}$ & $\begin{array}{l}\text { Año } \\
2015\end{array}$ & $\begin{array}{l}{ }^{*} \text { Tasa } \\
\text { Crec. } \\
15 / 14\end{array}$ & $\begin{array}{l}\text { Año } \\
2016\end{array}$ & $\begin{array}{l}{ }^{*} \text { Tasa } \\
\text { Crec. } \\
16 / 15\end{array}$ & $\begin{array}{l}\text { Año } \\
2017\end{array}$ & $\begin{array}{l}{ }^{*} \text { Tasa } \\
\text { Crec. } \\
17 / 16\end{array}$ & $\begin{array}{l}\text { Año } \\
2018\end{array}$ & $\begin{array}{l}\text { *Tasa } \\
\text { Crec. } \\
18 / 17\end{array}$ & $\begin{array}{l}\text { Año } \\
2019\end{array}$ & $\begin{array}{l}{ }^{*} \text { Tasa } \\
\text { Crec. } \\
19 / 18\end{array}$ \\
\hline Canadá & 1300092 & 110,60 & 1203430 & 92,60 & 1133824 & 94,20 & 1109630 & 97,87 & 1120077 & 100,94 \\
\hline $\begin{array}{l}\text { Estados } \\
\text { Unidos }\end{array}$ & 161233 & 176,70 & 281706 & 174,70 & 618346 & 219,50 & 639365 & 103,40 & 498538 & 77,97 \\
\hline $\begin{array}{l}\text { Comunidad } \\
\text { Cubana } \\
\text { Exterior }\end{array}$ & 390626 & 108,10 & 427228 & 109,40 & 517561 & 121,10 & 600306 & 116,00 & 623972 & 103,90 \\
\hline Alemania & 175264 & 126,00 & 242003 & 138,10 & 243172 & 100,50 & 208506 & 85,74 & 174956 & 83,91 \\
\hline Italia & 137727 & 122,90 & 191378 & 139,00 & 227829 & 119,00 & 208287 & 91,10 & 133436 & 64,06 \\
\hline Francia & 138474 & 133,80 & 187022 & 135,06 & 209239 & 111,88 & 177652 & 84,90 & 169394 & 95,35 \\
\hline Inglaterra & 155802 & 125,70 & 194618 & 124,90 & 168940 & 86,81 & 189813 & 81,40 & 123745 & 65,10 \\
\hline España & 107368 & 139,30 & 153340 & 142,80 & 141540 & 110,20 & 167969 & 80,90 & 146339 & 87,10 \\
\hline México & 105406 & 127,70 & 130881 & 124,20 & 209239 & 159,87 & 171945 & 82,18 & 166995 & 97,12 \\
\hline Rusia & 43387 & 62,70 & 65386 & 150,70 & 227829 & 161,00 & 137444 & 180,30 & 177977 & 129,50 \\
\hline Argentina & 85172 & 123,70 & 94727 & 111,20 & 99435 & 104,97 & 97358 & 97,91 & 95578 & 98,17 \\
\hline Total & 2800551 & 116,50 & 3171719 & 113,30 & 3796954 & 119,71 & 3708275 & 97,66 & 3431007 & 92,52 \\
\hline $\begin{array}{l}\text { Total } \\
\text { mercados }\end{array}$ & 3540175 & 117,50 & 4009169 & 113,20 & 4653559 & 116,10 & 4711910 & 101,10 & 4275558 & 90,70 \\
\hline $\begin{array}{l}\% \text { mercados } \\
\text { con relación } \\
\text { al total }\end{array}$ & 79,11 & & 79,11 & & 81,59 & & 78,70 & & 80,25 & \\
\hline
\end{tabular}

Nota. Las tasas de crecimientos entre los años, se presentan de forma porcentual. Fuente: Elaboración propia a partir de ONEI (2017, 2019a).

Dentro de la misma tónica del año anterior, el crecimiento alcanzado en el año 2016 (13,2 \% en llegadas de visitantes y $13,4 \%$ en llegada de turistas), también se encuentra entre los más altos del siglo XXI, donde EE.UU. continúa con un alto crecimiento (174\%) y existe un crecimiento vigoroso de los mercados principales, con la sola excepción de Canadá, que decrece un $7,4 \%$ por primera vez en muchos años en el arribo de sus nacionales a la Isla. También Rusia crece vigorosamente $(150,7 \%)$.

En el año 2017 se logra un crecimiento de 16,1\% en visitantes y 15,6 \% en turistas, uno de los más altos en la historia del turismo en Cuba. Encabeza el crecimiento EE.UU. con un estruendoso 219,5\%, impulsado también por el nuevo escenario relaciones entre Cuba y 
EE.UU., seguido por Rusia con un $161 \%$. Todos los demás mercados crecen: la Comunidad Cubana en el Exterior, los europeos (aunque con menor vigor que el año anterior) y los latinoamericanos. La única excepción es Canadá, que como el año anterior 2016, decrece, en este caso, en un $5,8 \%$.

El año 2018, sin embargo, no alcanza ni mantiene los ritmos de crecimiento de los años inmediatos anteriores y sólo logra un magro 1,1\% de crecimiento en las llegadas de visitantes y un $1,2 \%$ en la llegada de turistas. Entre las mayores influencias del tan bajo crecimiento de las llegadas, estuvo en primer lugar el menor crecimiento de las entradas procedentes del mercado estadounidense. Los estadounidenses que arribaban a partir de las categorías de viajes autorizados previamente, empezaron a disminuir con fuerza, luego de la restauración y fortalecimiento, en octubre de 2017, de las restricciones de los viajes a Cuba para los norteamericanos y la recomendación del nuevo gobierno de EE.UU. de no viajar a la Isla.

A esto siguió en marzo del propio año 2018, la retirada del personal diplomático de la embajada de EE.UU. en Cuba por afectaciones a la salud debido a supuestos ataques sónicos desconocidos, por lo que se declaraba que Cuba era un destino de riesgo para cualquier visitante. Otro factor se encontraba en la publicación de la "Cuba Restricted List", una larga lista de entidades estatales cubanas, con las cuales quedaba prohibido terminantemente a los norteamericanos hacer cualquier transacción financiera, esto con el propósito de que no se pudieran hacer pagos por servicios turísticos. Por las razones anteriores, en total en el 2018 las visitas de estadounidenses a Cuba, solo crecieron un modesto $3,4 \%$, el más bajo de todos los años anteriores.

En segundo lugar, en el año 2018, el tradicional mercado canadiense número uno para Cuba, continuó disminuyendo, en este caso ligeramente (2,2\%), aunque menos que los dos años anteriores, y en tercer lugar en forma significativa varios países europeos y Argentina, no vieron crecer sus emisiones a Cuba (contrario a lo que ocurrió en años anteriores). Para el caso de Reino Unido el proceso del Brexit, la baja de la libra esterlina y la inflación provocada, pudiera haber influido en la reducción de la emisión a Cuba y en general para los distintos países europeos, influyó también la quiebra de los turoperadores Thomas Cook (el 23 de septiembre) y de Holiday Place (que enviaban unos 95 mil turistas a Cuba anualmente), como también las repercusiones de la guerra comercial entre China y EE.UU.

Otro factor general que influyó en la reducción de visitas a Cuba en 2018 fue la percepción que se tenía tras la destrucción general que dejó el ciclón Irma, el cual azotó fuertemente al país a finales del 2017. 
Los crecimientos de Rusia y la Comunidad Cubana en el Exterior no compensaron los retrocesos y bajos crecimiento de la mayoría de los mercados principales, de ahí los reducidos crecimientos de visitantes y turistas en 2018.

En el año 2019, lo más novedoso y significativo resultó ser que en junio, se tomaría por el gobierno de los EE.UU. una medida más fuerte aún: se eliminaba la autorización de los viajes grupales pueblo a pueblo y de los viajes de cruceros, buques de pasajeros y recreativos y aviones privados y corporativos a Cuba (CIDTUR, 5 de junio 2019). Ello conllevó que, si hasta junio de 2019 existía un pequeño crecimiento de $0,1 \%$ de las llegadas de visitantes, a partir de julio todos los meses del año tuvieran retrocesos, por lo que las llegadas de visitantes del año 2019 fueran mucho menores que el año anterior.

En el caso de Canadá, dada la importancia de este mercado "las autoridades cubanas se plantearon para el 2019 alcanzar otra vez, la cuota de 1,3 millones" (Herrera, 2019), pero este país, al parecer influenciado por los mismos supuestos ataques sónicos, redujo a la mitad su personal diplomático en Cuba y el turismo solo logró crecer este año un 0,94 \%.

En 2019 Alemania, Francia, Italia, Inglaterra y España presentan una fuerte retracción de las emisiones a Cuba. México y Argentina también decrecen y solo la Comunidad Cubana en el Exterior y Rusia tienen crecimientos de sus llegadas en 2019 , junto a pequeño crecimiento de Canadá ya mencionado.

El año 2019, al cierre de diciembre, registró por primera vez en el lustro, un decrecimiento de 9,3\% al arribar a la isla 4275558 visitantes, que significaron 436352 menos que en igual período del año anterior, decrecimiento de la llegada de visitantes al país que solo había ocurrido durante los años de crisis de 2002, 2006 y 2007. El decrecimiento en turistas fue discretamente menor, un $9 \%$.

A estas medidas de EE.UU. para afectar la entrada de visitantes a Cuba - fuera del lustro analizado- se añadiría en enero de 2020 la no autorización de vuelos chárter públicos a Cuba excepto a La Habana, que afectaría a la Comunidad Cubana en el Exterior con origen de residencia en el resto de las provincias cubanas, la puesta en lista de países que no cooperan en la lucha contra el terrorismo a Cuba en mayo y la sucesiva ampliación de la lista de empresas cubanas con las cuales no pueden los estadounidenses tener ninguna relación financiera.

Así, de manera general entre 2015 y 2017 hay un crecimiento extraordinario de las llegadas de visitantes a Cuba, influido fuertemente por la emisión desde EE.UU. y en 2018 y 2019 los crecimientos bajan y retroceden las llegadas, determinados en gran medida por el mercado de 
EE.UU. y también por la baja considerable del resto de los principales mercados. Lo transcurrido en estos años pone de manifiesto el interés que existe en EE.UU. por el destino turístico Cuba.

En el panorama de 5 años de las llegadas de visitantes y turistas a Cuba, se debiera resaltar qué como promedio anual, crecieron ligeramente más los turistas $(5 \%)$, que los visitantes $(4,8$ \%) y específicamente en cada uno de los años, con excepción del 2017, los turistas crecieron más que los visitantes, todo lo cual es válido subrayar como algo positivo.

En el lustro 2015-2019 el crecimiento promedio de las llegadas a Cuba fue más alto (4,8 \% en llegadas de visitantes y $5 \%$ en turistas), que los crecimientos medios en el lustro anterior 2010 2014 (4,3\% en visitantes y 4,3\% en turistas) y que los crecimientos promedios de la primera década del siglo XXI ( $4 \%$ en visitantes y $4,1 \%$ en turistas).

Del análisis de los mercados emisores a Cuba, se destaca una observación tradicional, la alta concentración en pocos mercados, que se agudiza mucho más en el año 2017 (81,59 \% los 11 mercados principales) y en el 2019 con el 80,25 \% de las llegadas de visitantes.

Igualmente, en todo este periodo, las motivaciones de los viajes a Cuba que predominan son las de ocio y recreación, y sobre todo de sol y playa, asociados a los viajes programados. Por ejemplo, existe una contracción en una motivación distinta como la modalidad de eventos que en 2015 eran 3643 y en 2019 son 3117 (ONEI, 2019b), junto a otras modalidades como las de Salud, que desde 2014 la ONEI no las informa y que fueron significativas en periodos anteriores.

Sin embargo, en 2019 existe una moderada disminución de las motivaciones de ocio y recreación (93,4\%), con respecto al 2015 (95\%), que pudiera ser un atisbo del cambio de modelo de desarrollo turístico que se está produciendo, hacia uno más intensivo e inclusivo, con turismo de más altos ingresos, de ciudad, patrimonial, cultural y otros, complementarios al turismo de sol y playa.

\section{Comparaciones con el Caribe y el mundo.}

Podría ayudar a comprender el alcance del crecimiento de las llegadas a Cuba en los 5 años analizados, la comparación con las llegadas a los principales destinos receptores de turismo en el Caribe Insular, del Caribe Insular en su conjunto y del mundo, que se muestra en la tabla 2.

La única manera de comparar con otros países es a través de la llegada de turistas, según la estadística oficial que reporta la OMT de todos los países (no la llegada de visitantes).

Cuba, con las nuevas circunstancias de tener un mayor acceso al mercado de EE.UU. entre 2015 y 2017, se encontró en condiciones similares al resto de los grandes receptores turísticos del Caribe Insular, que han contado naturalmente con el fuerte mercado de EE.UU. 
Tabla 2

Llegadas de turistas Caribe y mundo 2015-2019 (miles)

\begin{tabular}{|c|c|c|c|c|c|c|}
\hline Destinos & 2015 & 2016 & 2017 & 2018 & 2019 & * CMA 2015-2019 \\
\hline República Dominicana & 5600 & 5959 & 6188 & 6569 & 6446 & $3,5 \%$ \\
\hline Puerto Rico & 3542 & 3736 & 3797 & 3068 & ND & $-4,0 \%$ \\
\hline Cuba & 3506 & 3975 & 4594 & 4684 & 4263 & $5,0 \%$ \\
\hline Jamaica & 2123 & 2181 & 2353 & 2473 & 2681 & $6,0 \%$ \\
\hline Bahamas & 1484 & 1482 & 1439 & 1634 & 1804 & $5,0 \%$ \\
\hline Caribe Insular & 24110 & 25143 & 26050 & 25806 & 27051 & $2,9 \%$ \\
\hline Mundo & 1189000 & 1235000 & 1332900 & 1408000 & 1462000 & $5,3 \%$ \\
\hline
\end{tabular}

Nota. CMA: Crecimiento medio anual. ND: no disponible. Fuente: Elaboración propia a partir de OMT (2019a y 2020).

En estas condiciones, Cuba crece 5 \% como promedio en el periodo 2015 - 2019 en llegadas de turistas, más que la República Dominicana (3,5\%) y más que el Caribe Insular (2,9\%), e iguala a Bahamas, mientras que crece menos que Jamaica (6\%) y ligeramente menos que el promedio del turismo mundial en este periodo $(5,3 \%)$.

Cuba, llegó a alcanzar el segundo lugar en el Caribe por llegadas de turistas en el 2016, lo mantiene hasta el 2018, y presumiblemente lo mantendrá también en el 2019, antecedido solo por República Dominicana.

\section{Visitantes a bordo de cruceros turísticos.}

Con la cifra record de 28983 pasajeros desembarcados en Cuba fundamentalmente por navieras europeas en 2015 , los cruceros habían tenido un notable crecimiento de $150 \%$, pero en 2016 se multiplicaron las llegadas de cruceristas. El presidente de EE.UU. Obama, en 2016, otorgó permisos de excepción al Departamento del Tesoro de Estados Unidos que permitieron la visita de cruceros a Cuba desde EE.UU. los que se unieron a los que ya llegaban desde Europa principalmente. El Adonia, de la compañía Fathom, filial de Carnival Cruises, salió de un puerto de Miami el 1 de mayo de 2016 con destino a La Habana, constituyéndose en el primer crucero autorizado a tocar un puerto cubano en más de 50 años. Como consecuencia de la incorporación de navieras de EE.UU. a visitar Cuba, en 2016 existió un crecimiento espectacular de 759 \%, con 219984 visitantes a bordo de cruceros que tocaron Cuba. En 2017 el crecimiento fue excelente (267\%) con 586710 cruceristas que pisaron tierra cubana.

En el año 2017 se aprobaron 17 compañías de cruceros, con 25 embarcaciones, 8 más que en la temporada anterior, y fueron programadas 265 escalas en Cuba. Este año, 23 líneas de 
cruceros realizaron 706 escalas en nueve destinos cubanos; de estas escalas, 304 en el puerto de La Habana, 145 en Cienfuegos y 104 en Santiago de Cuba, que son los principales puertos de cruceros del país (Perelló, 2018). Ello dio como resultado que para la temporada finalizada a mediados del 2018 se generaran más 600,000 pasajeros de cruceros.

Según la proyección del Consejo Comercial EEUU-Cuba (2017), los 286 viajes planificados que realizarían las tres compañías Norwegian Cruise, Carnival Cruise Line y Royal Caribbean a Cuba entre 2017 y 2019 transportarán 455,000 pasajeros y reportarán USD 623 millones para las navieras y USD 64 millones para Cuba debido a gastos de los cruceristas en transporte, excursiones, restaurantes y recuerdos. Solo en tasa portuaria pagarían a Cuba 19 millones de USD, tasa que se eleva mucho más cuando los buques son más grandes.

En 2018 se alcanzó la cifra de 850835 visitantes desembarcos de cruceros, el record histórico, y con un importante de crecimiento de $145 \%$ con respecto al periodo anterior.

Ese mismo año Cruise Critic, seleccionó a La Habana concluyendo el año 2018, como mejor destino y puerto de cruceros en el Caribe occidental y la Riviera Maya. De acuerdo a lo anterior, y por la altísima demanda de visitar Cuba a bordo de los cruceros, se incorporó la Regent Seven Seas Cruises, naviera norteamericana de lujo para realizar viajes a Cuba a partir de 2019 y 2020.

En el año 2018 operaban con llegadas a Cuba, las compañías de EE.UU. Norwegian, Royal, Carnival, Pearl Seas y Columbia Cruise. De otros países operaban Italia-Suiza: MSC (ItaliaSuiza), Inchcape Shipping (Alemania) y Viking Cruises (Suiza).

Sin embargo, en junio de 2019 se prohíbe por el gobierno de Donald Trump la autorización de los viajes de cruceros, buques de pasajeros y recreativos y aviones privados y corporativos a Cuba. Según la Asociación Internacional de Líneas de Cruceros (CLIA), casi 800 mil reservas de cruceros se vieron afectadas por la decisión del Gobierno de Estados Unidos de prohibir, "sin advertencia alguna", los viajes a Cuba. Ello provocó enormes pérdidas a las navieras e ingresos dejados de obtener por Cuba. "La compañía Norwegian Cruise pronosticó una caída de hasta un ocho por ciento de beneficios por acción en 2019 por la decisión del gobierno de los EE.UU. de suspender los viajes de cruceros hacia Cuba" (CIDTUR, 11 de junio 2019).

No obstante, aunque en mucha menor medida, otros cruceros arribaron a Cuba, como por ejemplo el arribo a La Habana a fines de octubre del buque alemán Hamburg, el cual visitó después Cayo Largo, María la Gorda, Cienfuegos, Trinidad y Santiago de Cuba (Martínez, 2019), o el buque Panorama, de bandera griega, perteneciente a la compañía Variety Cruises, con 
capacidad para medio centenar de pasajeros, que arribó al puerto de Casilda, en Trinidad el 18 de noviembre de 2019 (CIDTUR, 19 de noviembre 2019).

Finalmente, como consecuencia de las medidas del gobierno de EE.UU. en 2019 se redujo a 422863 la cantidad de visitantes a bordo de cruceros, de ellos 365116 turistas, por lo que retrocedieron un 50 \% las llegadas de cruceristas con respecto al 2018.

Aunque es preferible un crecimiento mayor de turistas que de visitantes y cruceristas, en el caso de Cuba, que no ha podido contar con un desarrollo de los cruceros como el resto del Caribe, la potenciación exponencial del crecimiento de crucero en el periodo analizado indudablemente resultó beneficiosa (figura 2).

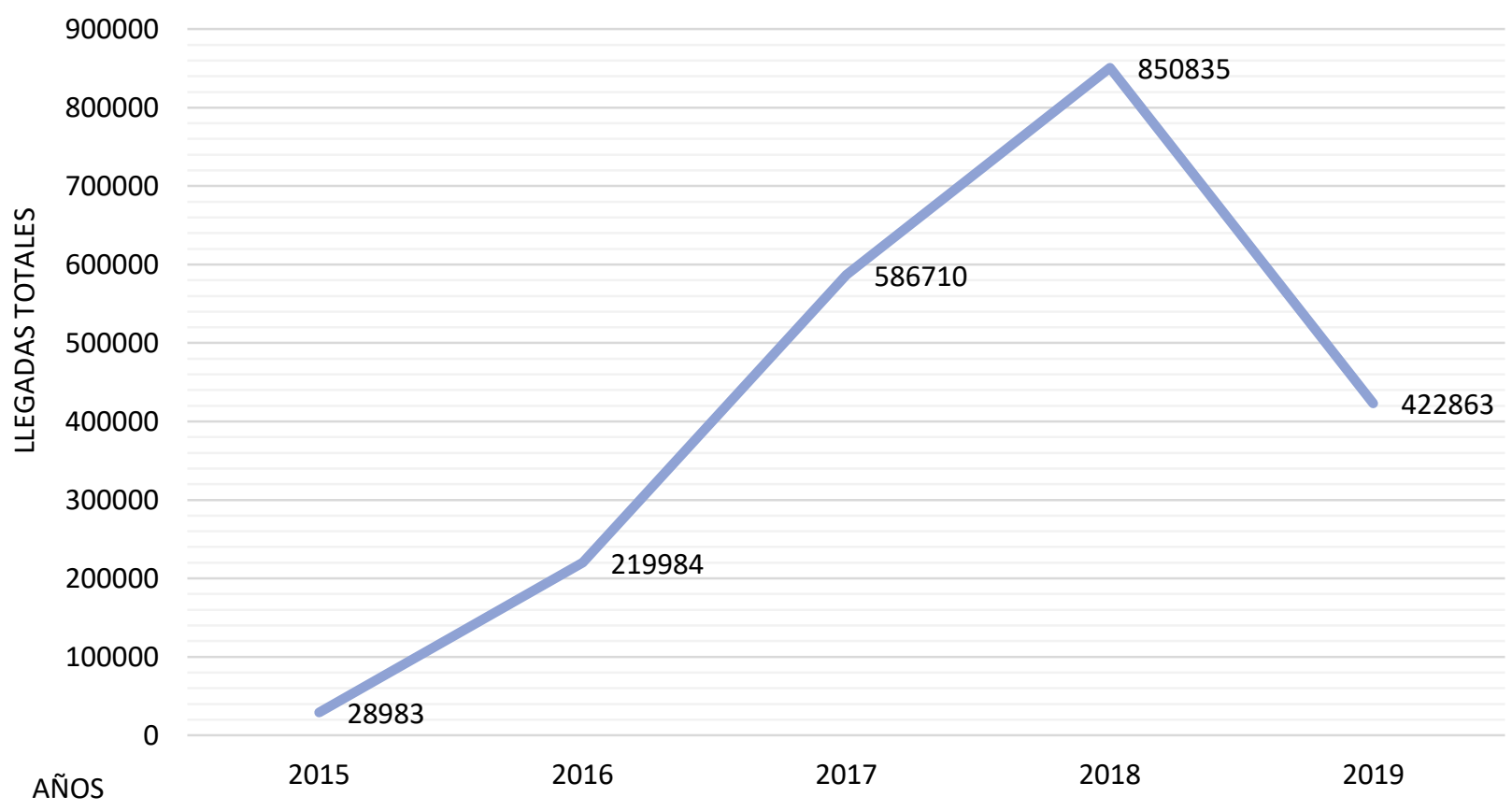

Figura 2. Pasajeros de Cruceros desembarcados en Cuba. 2015-2019.

Fuente: Elaboración propia a partir de Ministerio de Turismo, MINTUR (2020).

La fuente de información de los pasajeros de cruceros es del MINTUR, debido a que las informaciones que brindaba la ONEI no se correspondían con la realidad y el Anuario Estadístico 2019 ya dejó de informar el número de pasajeros de cruceros dentro de los visitantes internacionales.

Las agencias de viajes que en tierra atienden a los pasajeros de cruceros, como Havanatur, Cubanacán y Cubatur, vieron afectados sustancialmente sus ingresos en 2019 con la prohibición de visitar Cuba a los cruceros de EE.UU. y en particular los trabajadores por cuenta propia sufrieron una enorme pérdida al no contar con clientes que habitualmente reclamaban sus servicios. 


\section{Ingresos asociados al turismo internacional.}

El crecimiento de los ingresos asociados al turismo internacional en Cuba del 2015 (10,7 \%), del 2016 (8,9 \%) y del 2017 (8,0 \%), multiplican el crecimiento medio anual 2001 - 2014 (2,5\%), lo cual representó crecimientos que sobrepasaban lo habitual. Lo mismo ocurre con los ingresos turísticos propiamente dichos del 2015 al 2017, que presentan crecimientos nunca vistos en el presente siglo. Es incuestionable la influencia del mercado de EE.UU. en este periodo, que además es un mercado altamente gastador. Los principales indicadores del lustro 2015 - 2019 relacionados con los ingresos se presentan en la tabla 3.

Tabla 3

Principales indicadores relacionados con los ingresos asociados al turismo internacional: Cuba 2015-2019

\begin{tabular}{|c|c|c|c|c|c|c|c|c|c|c|c|}
\hline $\begin{array}{c}\text { Concepto: } \\
\text { Millones pesos } \\
\text { convertibles }\end{array}$ & 2015 & $\begin{array}{c}\% \\
15 / 14\end{array}$ & 2016 & $\begin{array}{c}\% \\
16 / 15\end{array}$ & 2017 & $\begin{array}{c}\% \\
17 / 16\end{array}$ & 2018 & $\begin{array}{c}\% \\
18 / 17\end{array}$ & 2019 & $\begin{array}{c}\% \\
19 / 18\end{array}$ & $\begin{array}{c}\% \\
* \text { CMA } \\
15 / 19\end{array}$ \\
\hline \multicolumn{12}{|l|}{ Ingresos } \\
\hline $\begin{array}{l}\text { Asociados Turismo } \\
\text { Internacional }\end{array}$ & 2818,6 & 110,7 & 3068,6 & 108,9 & 3301,7 & 108,0 & 2782,2 & 84,3 & 2645,1 & 95,1 & -1 \\
\hline $\begin{array}{l}\text { Ingresos } \\
\text { turísticos }\end{array}$ & 2600,8 & 109,9 & 2907,1 & 111,8 & 3185,9 & 110,0 & 2702,6 & 91,1 & 2596,2 & 96,1 & -0 \\
\hline $\begin{array}{l}\text { Ingresos sector } \\
\text { no estatal }\end{array}$ & 485,2 & 128,0 & 537,3 & 111,0 & 706,6 & 132 & 473,9 & 67,1 & 373,1 & 78,7 & -6 \\
\hline
\end{tabular}

Nota. CMA: Crecimiento medio anual. Fuente: ONEI (2019b).

En el año 2018 como consecuencia del reducido crecimiento de las llegadas de visitantes, los ingresos asociados al turismo internacional retrocedieron un 15,7\%, y los ingresos turísticos un 8,9 \%. En el año 2019 los ingresos asociados al turismo internacional se reducen en 4,9 \% y los ingresos turísticos en 3,9\%. El sector no estatal fue afectado más fuertemente: en el año 2018 $(32,9 \%)$ y en el $2019(21,3 \%)$ (Figura 3). 


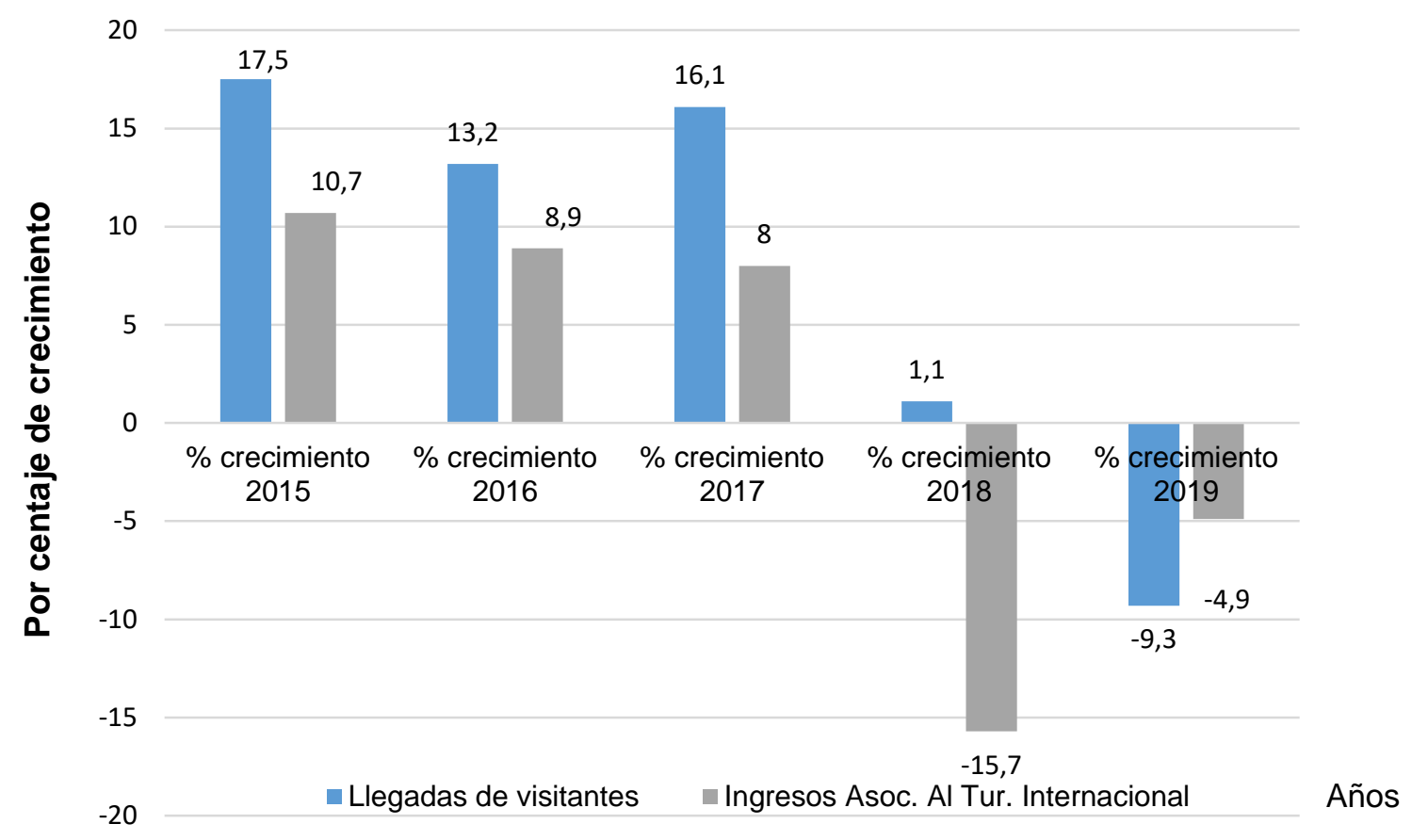

Figura 3. Comparación de los crecimientos de las llegadas de visitantes y los ingresos asociados al turismo internacional.

Fuente: Elaboración propia a partir de ONEI (2019b).

Al comparar los crecimientos de las llegadas y los ingresos, se observa como regla que las llegadas crecen más que los ingresos en 2015, 2016 y 2017, tal y como ha venido ocurriendo en todo el siglo XXI; en 2018 crecen poco las llegadas, y bajan notablemente los ingresos, mientras que en 2019 bajan más las llegadas que los ingresos, lo que representa un indicador favorable en este año, específicamente: se atienden menos turistas y se recaudan más ingresos relativamente.

En relación con los ingresos de las entidades turísticas (Tabla 4), se elevaron en 2015 (10,7 \%) muy por encima de los resultados de crecimiento de años anteriores, por lo que se multiplicó por poco más de 3 veces el promedio de crecimiento 2010 - 2014 (3\%). Todos los sectores crecen en 2015, menos la Recreación. El año 2016 completo no se encuentra disponible la publicación.

En 2017 se observa un crecimiento general, pero no existe forma de compararlo. En 2018 los ingresos de las entidades turísticas se reducen al 95,4 \% del año anterior y sectores claves como alojamiento, gastronomía y transporte automotor retroceden también. 
Durante el 2019 se presenta un decrecimiento moderado (99,7 \%), sobre el deprimido año anterior, y presentan reducciones absolutas sectores importantes como alojamiento, transporte y recreación.

Es lógico que decrezcan los ingresos por alojamiento, si se tiene en cuenta que según otra publicación de la ONEI (2019b), la tasa ocupación desde 2017 muestra decrecimiento: 42,5\% en $2017,38,5 \%$ en 2018 y $36,9 \%$ en 2019 .

Tabla 4

Principales indicadores de ingresos entidades turísticas 2015-2019

\begin{tabular}{|c|c|c|c|c|c|c|c|c|c|}
\hline $\begin{array}{c}\text { Concepto en } \\
\text { Millones de pesos } \\
\text { convertibles }\end{array}$ & 2015 & $\begin{array}{c}\% \\
15 / 14\end{array}$ & 2016 & 2017 & 2018 & $\begin{array}{c}\% \\
18 / 17\end{array}$ & 2019 & $\begin{array}{c}\% \% \\
19 / 18\end{array}$ & $\begin{array}{c}\% \\
* \text { CMA } \\
15 / 19\end{array}$ \\
\hline Ingresos entidades turísticas & 1940,2 & 110,7 & ND & 2296,9 & 2191,9 & 95,4 & 2184,6 & 99,7 & 3 \\
\hline Alojamiento & 558,7 & 106,5 & ND & 698,9 & 622,8 & 89,1 & 571,1 & 91,7 & 0,5 \\
\hline Comercio minorista & 185 & 109,3 & ND & 246,2 & 272,2 & 110,6 & 274,3 & 100,8 & 10 \\
\hline Gastronomía & 755,7 & 110,5 & ND & 891,9 & 857,4 & 96,3 & 897,8 & 104,7 & 4,3 \\
\hline Transporte automotor & 345,6 & 122,9 & ND & 279,5 & 186,8 & 66,8 & 177,3 & 94,9 & -15 \\
\hline Recreación & 19,2 & 112,7 & ND & 35 & 39,3 & 112,3 & 38,5 & 97,9 & 19 \\
\hline Otros & 66,9 & 97,2 & ND & 145,3 & 213,3 & 146,8 & 225,6 & 105,8 & 35 \\
\hline
\end{tabular}

Nota. *CMA: Crecimiento medio anual. ND: no disponible. Fuente: ONEI (2015, 2018, 2019b).

Se observa, no obstante, que la tasa de ocupación que presenta el Anuario Estadístico de Cuba de los medios de alojamiento del turismo es distinta, aunque se corresponde con la tendencia a la baja: 2017 (56,7\%), 2018 (49,5\%) y 2019 (48,2\%). Es necesario observar que la actividad turística ve afectada la eficiencia por los niveles de ocupación bajos, como en el caso del 2018 (Rodríguez, 2019).

En conjunto, para el lustro 2015 - 2019, el crecimiento promedio de los ingresos de las entidades turísticas (3\%) se iguala con el crecimiento obtenido en el quinquenio anterior 2010 2014. 
A continuación, en la figura 4, se muestra el ingreso asociado al turismo internacional por visitante y el ingreso turístico por turista de Cuba, en el periodo 2014 - 2019.

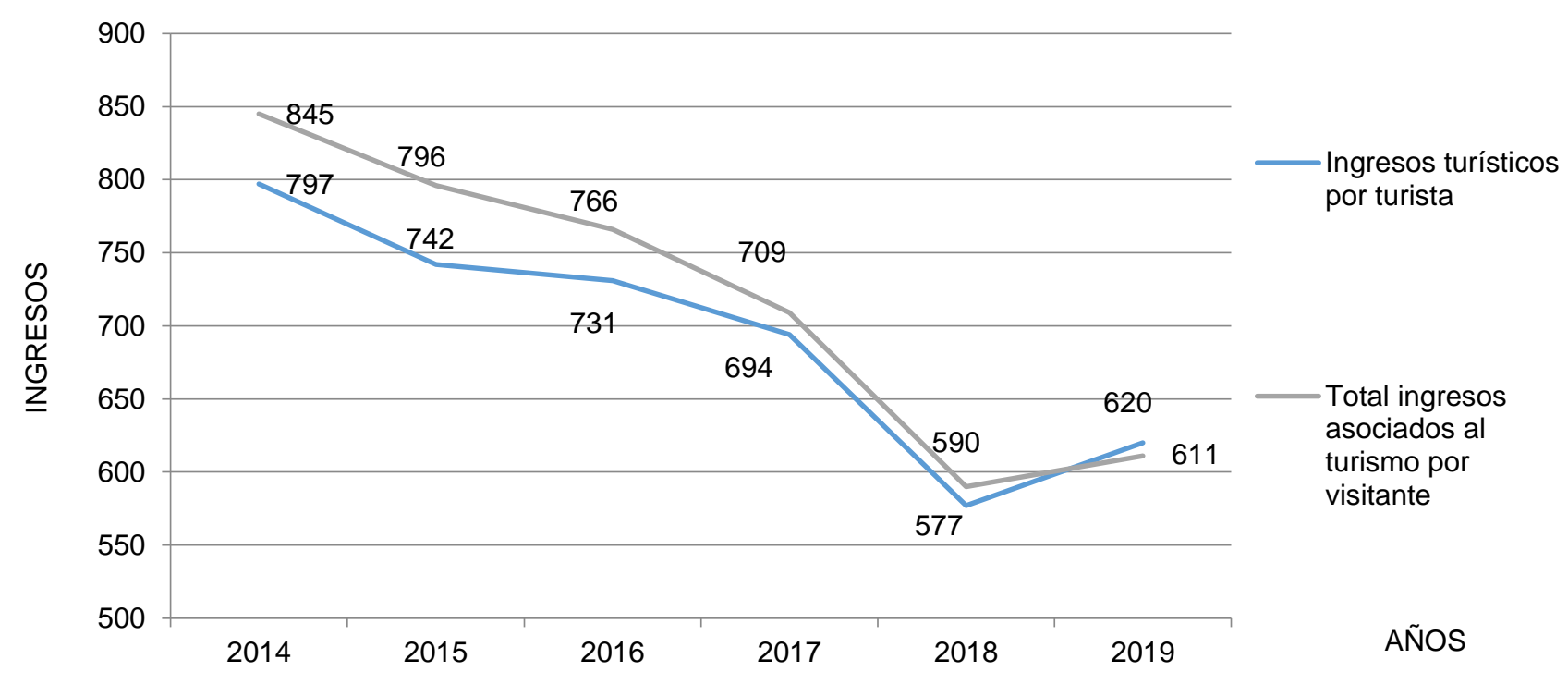

Figura 4. Ingresos por visitantes y turistas en Cuba 2014-2019.

Fuente: Elaboración propia a partir de ONEI (2019b).

Los ingresos asociados al turismo internacional por visitantes en el lustro 2014 - 2018 y los ingresos turísticos por turista continúan la tendencia decreciente que presentaban desde el año 2007, cuando específicamente el ingreso asociado al turismo internacional por visitante de Cuba alcanzó el record de 1110 USD (Ayala, 2013).

En 2019 se presenta un cambio de tendencias que es favorable. Tanto el total de ingresos asociados al turismo internacional por visitante, como el ingreso turístico por turista suben, por lo que se alcanza por primera vez un valor más alto el ingreso por turista que el ingreso asociado al turismo por visitante. A partir de ello, es necesario estudiar si este cambio se corresponde con alguna modificación del tipo de turismo que se recibió en 2019, pues no existen estudios que permitan interpretarlo de esta manera.

Los datos reflejados en la tabla 5 permiten establecer una comparación entre los ingresos turísticos en el periodo 2015 - 2019 de Cuba, y de otros importantes receptores del Caribe Insular, del Caribe Insular en su conjunto y el mundo.

Cuba en el periodo 2015 - 2019 en cuanto a ingresos turísticos, como promedio no crece; mientras que el mundo, el Caribe, República Dominicana y Bahamas crecen. La excepción es Puerto Rico, que no está publicada su información todavía por la OMT y que fue afectado por un devastador huracán en 2018, cuyos efectos aún repercuten en el turismo. 
Cuba mantuvo el tercer lugar por ingresos turísticos en el Caribe en el 2017, pero en el 2018 retrocedió a quinto lugar, antecedido por República Dominicana, Bahamas, Puerto Rico y Jamaica, y presumiblemente en el 2019 sostenga esta posición.

Tabla 5

Millones de USD de ingresos turísticos de principales destinos del Caribe y el mundo

\begin{tabular}{|c|c|c|c|c|c|c|c|c|}
\hline Destinos & 2014 & 2015 & 2016 & 2017 & 2018 & 2019 & $\begin{array}{c}{ }^{*} \mathrm{CMA} \\
2014- \\
2018\end{array}$ & $\begin{array}{l}{ }^{*} \text { CMA } \\
2015- \\
2019\end{array}$ \\
\hline $\begin{array}{l}\text { República } \\
\text { Dominicana }\end{array}$ & 5630 & 6116 & 6720 & 7178 & 7561 & 7468 & $7,6 \%$ & $5,1 \%$ \\
\hline Puerto Rico & 3439 & 3825 & 3974 & 3848 & 3282 & $* N D$ & $-1 \%$ & - \\
\hline Cuba & 2367 & 2601 & 2907 & 3186 & 2703 & 2596 & $3,3 \%$ & $-0 \%$ \\
\hline Jamaica & 2255 & 2401 & 2539 & 2809 & 3099 & *ND & $8,2 \%$ & - \\
\hline Bahamas & 2308 & 2537 & 2591 & 2577 & 3355 & 3580 & $9,8 \%$ & $8,9 \%$ \\
\hline Caribe Insular & 26688 & 29336 & 30773 & 31795 & 32706 & 35934 & $5,2 \%$ & $5,2 \%$ \\
\hline Mundo & 1252000 & 1202000 & 1247000 & 1350000 & 1454000 & 1480000 & $3,8 \%$ & $5,3 \%$ \\
\hline
\end{tabular}

Nota. CMA: Crecimiento Medio Anual. ND: no disponible. Fuente. Elaboración propia a partir de OMT (2019a y 2019b) y ONEI (2019a).

Al comparar el ingreso turístico por turista en el periodo de 2015 - 2019 (Tabla 6), con los otros grandes destinos receptores del Caribe, el Caribe Insular en su conjunto y el Mundo, se pone de manifiesto que no necesariamente es una tendencia universal que decrezcan los ingresos turísticos por turista con el paso del tiempo. Se observa que Cuba muestra una tendencia decreciente en el periodo, es decir los ingresos por turista cada año son más bajos (con excepción del 2019 pero que es más bajo que el 2017). Lo mismo pasa con Puerto Rico, Bahamas y el Mundo, pero República Dominicana, Jamaica y el Caribe Insular en su conjunto muestran una tendencia de crecimiento.

Con los resultados reflejados, se puede afirmar que, Cuba destaca por poseer el indicador cuantitativo más bajo absoluto en cada año del periodo analizado en comparación con los principales destinos receptores del Caribe insular, el Caribe insular y el mundo.

Los autores, llaman la atención sobre este aspecto, a los efectos de que se considere la necesidad de revertir la tendencia que muestra; en tanto se continúan investigando las causas, que podrían ser, entre otras, que Cuba se ha encontrado demasiado concentrada en el producto sol y playa, y muy poco en otras modalidades turísticas más favorables. 
De igual manera, puede incidir sobre estos números que los mercados habituales que visitan el destino, y con los cuales se trabaja no son los mercados más gastadores, y que el destino aún no posee una cantidad suficiente de atractivos turísticos comercializados por extra hoteleros, para el consumo de los turistas.

Tabla 6 Ingresos turísticos por turista: Caribe Insular y mundo

\begin{tabular}{lcccccc}
\hline \multicolumn{1}{c}{ Destinos } & 2014 & 2015 & 2016 & 2017 & 2018 & 2019 \\
\hline República Dominicana & 1,095 & 1,092 & 1,128 & 1,160 & 1151 & 1159 \\
Puerto Rico & 1,079 & 1,080 & 1,067 & 1,077 & 1070 & *ND \\
Cuba & 797 & 742 & 731 & 694 & 577 & 620 \\
Jamaica & 1,084 & 1,131 & 1,164 & 1,194 & 1253 & *ND \\
Bahamas & 2,153 & 1,710 & 1,748 & 1,791 & 2055 & 1984 \\
Caribe Insular & 1,197 & 1,217 & 1,224 & 1,214 & 1246 & 1328 \\
Mundo & 1,101 & 1,011 & 1,010 & 1,008 & 1034 & 1012 \\
\end{tabular}

Nota. 1ND: no disponible. Fuente: Elaboración propia a partir de OMT (2019a y 2020).

\section{CONCLUSIONES}

El estudio realizado permite afirmar que, para todo el lustro, aún con años en que no existieron resultados positivos, el crecimiento promedio de las llegadas de visitantes fue superior al crecimiento promedio del lustro anterior, y de la primera década del siglo XXI, lo cual resulta positivo. Otro elemento favorable ha sido que el crecimiento de los turistas es mayor que el crecimiento de los visitantes.

De igual forma, se puede enunciar que bajo las nuevas circunstancias de mayor acceso al mercado de EE.UU. desde 2016, Cuba llega a ostentar el segundo lugar en llegada de turistas en el Caribe Insular, y en el periodo 2015 - 2019 crece como promedio en llegadas turísticas más que República Dominicana y el Caribe Insular en su conjunto, igualándose al crecimiento turístico de Bahamas, por lo que se corrobora que, de existir la posibilidad de viajar libremente a Cuba desde EE.UU. el número de arribos continuaría en ascenso. Se evidencia que, en cuanto a la actividad de cruceros, los ingresos monetarios dejados de percibir en Cuba por la interrupción de estas actividades a mediados de 2019 por parte del gobierno de EE.UU., luego de una apertura anterior, son cuantiosos. De igual forma, la continuidad de llegada de cruceros de compañías europeas, indica la conveniencia de continuar el desarrollo de la gestión de estas operaciones. 
El crecimiento de los ingresos asociados al turismo internacional en Cuba entre 2015 y 2017 , multiplica por varias veces el crecimiento medio anual de todos los años precedentes (2001 2014) y los crecimientos de los ingresos turísticos propiamente dichos, también rompen record en el siglo XXI. Pero, como resultado de la retracción del mercado de los EE.UU. principalmente, los ingresos asociados al turismo internacional y los ingresos turísticos son negativos en 2018 y 2019.

De forma general, Cuba en el periodo 2015 - 2019 en cuanto a ingresos turísticos, como promedio anual no crece, a diferencia del resto de los destinos principales del Caribe, el Caribe en su conjunto y el mundo, mientras que el ingreso por turista en Cuba, entre 2015 y 2019, ha sido el indicador cuantitativo más pequeño existente entre los principales destinos receptores del Caribe insular, del Caribe insular en su conjunto y de la media de todo el mundo.

\section{REFERENCIAS BILIOGRÁFICAS}

Ayala, H. (2013). Ingresos asociados al turismo en Cuba en la primera década del siglo XXI. Revista Caribeña de Ciencias Sociales, 1-9.

Ayala, H. (2016). Tendencias de los ingresos turísticos de Cuba en el periodo 2010 - 2014. Retos Turísticos, $15(1), 1-12$.

Consejo Comercial EEUU-Cuba (2017). Los cruceros con Cuba generan $540 \mathrm{M} €$ para las navieras en tres años. Recuperado de: https://www.hosteltur.com/125017_cruceroscuba-generan-540-m-navieras-tres-anos.ht

Herrera, H. (2019). Breves notas para un turismo resiliente en Cuba. Recuperado de: https://cubayeconomia.blogspot.com/2019/05/breves-notas-para-un-turismoresiliente.html

Hernández, Y. (2020). Diseño del escenario más probable de la OSDE Viajes Cuba al año 2015. (Tesis de Licenciatura). Facultad de Turismo, Universidad de La Habana, Cuba.

Hernández, R., Fernández, C. y Baptista, M. (2014). Metodología de la investigación. Sexta Edición. México: Mc Graw Hill Education.

Kieffer, M. (2018). Conceptos claves para el estudio del turismo rural comunitario. El periplo sustentable, (37), 8-43.

Martínez, Y. (2019). Buque alemán MS Hamburgo ancla en La Habana: Inicia temporada de cruceros procedentes de Europa. Recuperado de: http://www.cubadebate.cu/noticias/2019/10/30/buque-aleman-ms-hamburgo-ancla-en-lahabana-inicia-temporada-de-cruceros-procedentes-de-europa-video/ 
Ministerio de Turismo (2020). Llegadas de cruceristas al destino Cuba 2015 - 2019. La Habana: MINTUR.

Oficina Nacional de Estadísticas e Información. (2015). Turismo Internacional. Indicadores seleccionados enero - diciembre 2015. Recuperado de: https://www.onei.gob.cu

Oficina Nacional de Estadísticas e Información. (2017). Anuario Estadístico de Cuba 2016. Turismo. Recuperado de: https://www.onei.gob.cu

Oficina Nacional de Estadísticas e Información. (2018). Turismo Internacional. Indicadores seleccionados enero - diciembre 2018. Recuperado de: https://www.onei.gob.cu

Oficina Nacional de Estadísticas e Información. (2019a). Anuario Estadístico de Cuba 2018. Recuperado: https://www.onei.gob.cu

Oficina Nacional de Estadísticas e Información. (2019b). Turismo Internacional. Indicadores seleccionados enero - diciembre 2019. Recuperado de: https://www.onei.gob.cu

Organización Mundial del Turismo (2014). Panorama OMT del turismo internacional. Edición 2014. Recuperado de: http://www.e-unwto.org/

Organización Mundial del Turismo (2019a). Panorama OMT del turismo internacional. Edición 2019. Recuperado de: http://www.e-unwto.org/

Organización Mundial del Turismo (2019b). Barómetros OMT del turismo mundial 2019. Recuperado de: http://www.e-unwto.org/

Organización Mundial del Turismo (2020). Barómetros OMT del turismo mundial Enero Julio 2020. Recuperado de: http://www.e-unwto.org/

Perelló, J. L. (2018). El turismo de cruceros en Cuba y La Habana. Recuperado de: https://www.excelenciascuba.com/noticia/el-turismo-de-cruceros-en-cuba-y-la-habana

CIDTUR (5 de junio de 2019). EE.UU. prohíbe los cruceros a Cuba en un nuevo paquete de Trump que restringe aún más los viajes a la isla. Reportes de prensa.

CIDTUR (11 de junio 2019). Norwegian Cruise confirma pérdidas millonarias por medidas de Trump. Reportes de prensa.

CIDTUR (19 de noviembre 2019). Inicia temporada alta de arribo de cruceros a Trinidad. Reportes de prensa.

Rodríguez, J. L. (2019). Balance preliminar de la economía cubana en el 2018 y algunas perspectivas para el 2019 (III). Recuperado de: http://clif.in/2019/03/04/balanceeconomico-preliminar-del-2018-en-cuba-y-algunas-perspectivas-para-el-2019/ 